\title{
Characterization of Cytotoxic T Lymphocytes Recognizing Autologous Tumor Cells with Plural HLA-class I Alleles in a Patient with Esophageal Cancer
}

\author{
TOMOYUKI MAEDA \\ Department of Immunology, Kurume University School of Medicine, \\ Kurume 830, Japan
}

Received for publication December 5, 1996

\begin{abstract}
Summary: T cell sublines and clones were established from the parental HLA-A2601-restricted cytotoxic $\mathrm{T}$ lymphocyte line (KE-4 CTL, HLAA2601/2402, -B5101/40012, -Cw0102/0302) to investigate their capability to recognize tumor antigens on HLA-class I alleles other than HLA-A2601. Thirty-nine of $52(75 \%)$ sublines or 30 of $83(36 \%)$ clones produced interferon (IFN) $-\gamma$ by recognition of the autologous KE- 4 tumor cells. Among these 39 CTL sublines, 22,22 , and 2 sublines produced IFN- $\gamma$ by recognition of HLA$\mathrm{A} 2402^{+},-\mathrm{B}_{101}{ }^{+}$, and $\mathrm{Cw} 0102^{+}$allogenic tumor cells, respectively. Four, 8, and 4 of these 30 CTL clones also produced IFN- $\gamma$ by recognition of HLA$\mathrm{A} 2402^{+},-\mathrm{B} 5101^{+}$, and $\mathrm{Cw} 0102^{+}$allogenic tumor cells, respectively. The other 13 sublines or 53 clones failed to produce IFN- $\gamma$ by recognition of the autologous tumor cells, respectively. These results suggest the presence of CTL recognizing shared tumor antigens on autologous tumor cells expressed by plural HLA-class I alleles in a patient with esophageal SCC.
\end{abstract}

Key words HLA-class I alleles, cytotoxic T lymphocytes, squamous cell carcinomas

\section{Introduction}

Squamous cell carcinoma (SCC) is one of the most common cancers in human. SCC, particularly, esophageal and lung SCC, are relatively resistant currently available regimens of chemotherapy or radiation therapy. Therefore, development of a specific immunotherapy using tumor specific cytotoxic $\mathrm{T}$ lymphocytes
(CTL) would be important to offer other treatment modalities. Tumor specific CTL have been generated in vitro from peripheral blood mononuclear cells (PBMC) or tumor-infiltrating lymphocytes of patients with melanomas and ovarian cancers (Itoh et al. 1988; van der Bruggen et al. 1991; Ioannides et al. 1993; Peoples et al. 1993; Coulie et al. 1994; Kawakami et al. 1994a,b). Genes encoding

Supported in part by a Grant-in-Aid for Scientific Research from the Ministry of Education, Science and Culture, Japan, a grant from the Science Research Promotion Fund of Japan Private School Promotion Foundation, and a grant from Fukuoka Cancer Society, Fukuoka, Japan.

Correspondence should be addressed to: T. Maeda, Department of Immunology, Kurume University School of Medicine, 67 Asahi-machi, Kurume 830, Japan. Tel: 81-942-31-7551 Fax: 81-942-31-7699 
tumor-rejection antigens were also identified using these CTL lines and clones from melanoma patients (van der Bruggen et al. 1991; Coulie et al. 1994; Kawakami et al. 1994a,b). These studies showed that CTL in a melanoma patient recognize many different antigens of autologous tumor cells expressed on plural HLA-class I alleles (Boon et al. 1995). We have recently established the HLA-A2601-restricted CTL line recognizing a peptide antigen expressed on SCC in PBMC of an esophageal patient (Nakao et al. 1995). The present study investigated whether this parental CTL line possessed $\mathrm{T}$ cells recognizing antigens of autologous tumor cells with HLA-class I alleles other than A2601 allele. The results showed the presence of the CTL recognizing antigens of autologous tumor cells expressed on plural HLA-class I alleles in a patient with esophageal SCC.

\section{Materials and Methods}

\section{A patient, CTL line and tumor cell lines}

The KE-4 tumor cell line was established from a patient with esophageal cancer in our hospital (Nakao et al. 1995). PBMC were isolated for the generation of CTL. The HLA-A2601restricted and tumor-specific CTL (KE-4 CTL) were established by the repeated stimulation of PBMC with autologous KE-4 tumor cells, and the detailed characterization was previously reported (Nakao et al. 1995). Tumor cell lines used in this study and their genotypes of HLA-class I alleles were determined by polymerase chain reaction-sequence specific oligonucleotide probe (PCR-SSOP) method as reported (Nakao et al. 1995; Seki et al. 1997).

Generation and Culture of CTL
sublines and clones

CTL sublines were established from the parental KE-4 CTL line by incubation of different numbers of cells $(128,64,32$, $16,8,4,2$, and 1) per well of U-bottom 96-well microplate in $0.2 \mathrm{ml}$ of the medium (50\% RPMI 1640 medium and 50\% AIM-V medium) supplemented with $10 \%$ FCS, $100 \mathrm{U} / \mathrm{ml}$ rIL-2, $10 \mu \mathrm{g} / \mathrm{ml}$ PHA-P (Difco Laboratories, Gland Island, NY), 1\% T-STIM $^{\mathrm{TM}}$ with PHA (Becton Dickinson Labware, Bedford, MA) in the presence of $2 \times 10^{5}$ cells/well of irradiated allogenic PBMC from 3 different healthy donors. For establishment of $\mathrm{T}$ cell clones, 0.5 cells per well of the parental CTL line were incubated with the same condition shown above. The culture medium was changed with the medium mentioned above in the absence of allogenic PBMC every 7 days and the proliferating cells were transferred into a well of 48 wellculture plate around 21 days of culture. The cells were transferred into a well of 24 well-culture plate around 28 days of culture followed by further expansion. The surface phenotype of the cells was studied by a direct immunofluorescence assay with FITC-conjugated anti-CD3, -CD4, and -CD8 (Nichirei, Tokyo) monoclonal antibody (mAb) and FACScan (Becton-Dickinson, Mountain View, CA) (Nakao et al. 1995), and only $\mathrm{CD}^{+} \mathrm{CD} 4^{-}$ $\mathrm{CD}^{+} \mathrm{T}$ cells were used as potential CTL sublines and clones in this study.

Interferon gamma (IFN- $\gamma)$ production

IFN- $\gamma$ production by the CTL sublines and clones in response to tumor 

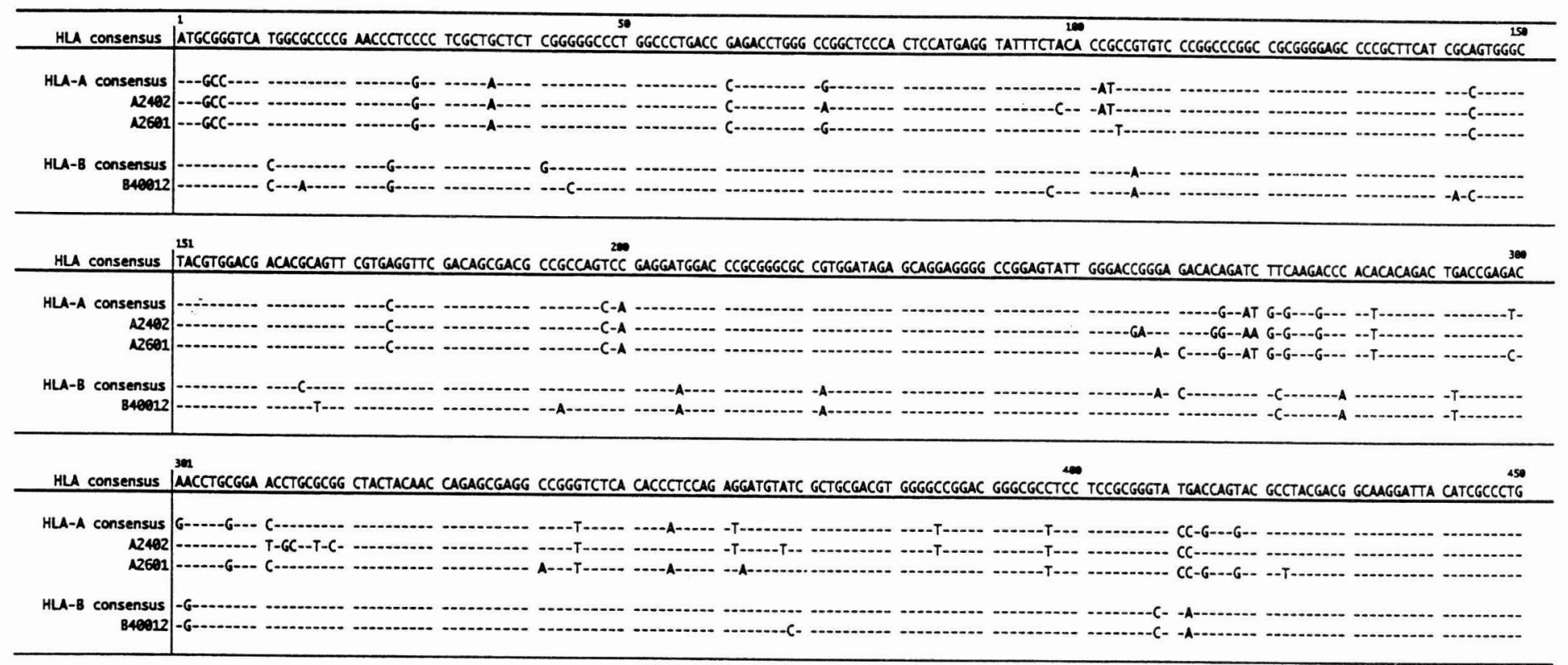

Fig. 1. Nucleotide sequences of HLA-class I alleles of the KE-4 patient: Ten million cells of either the KE-4 CTL or KE-4 tumor cells were used to prepare mRNA using a Quick Prep mRNA Purification Kit (Pharmacia, Uppsala, Sweden), and firststrand cDNA was synthesized using a Super Script Preamplification Kit (BRL, Gaithersburg, MD). HLA-A specific oligonucleotides (5'-primer; GAATCTCCCCAGACGCCGA, 3'-primer; TGTCTCACACTTTACAAGCTGTGAGAG) and PCR were used to amplify HLA-A cDNA. HLA-B specific oligonucleotides (5'-primer; GAATCTCCCCAGACGCCGA, 3'-primer; AAACACAGGTCAGCATGGGAAC) or HLA-C specific oligonucleotides (5'-primer; GAATCTCCCCAGACGCCGA, 3'-primer; GAACACAGGTCAGTGTGGGG) and PCR were used to amplify HLA-B or -C cDNA, respectively. These HLA-cDNA were cloned using an Eukaryotic TA Cloning Kit (Invitrogen, San Diego, CA) according to the manufacturer's instruction, and were sequenced using an A.L.F. DNA Sequencer (Pharmacia) as reported (Nakao et al. 1995). The part of the sequences were shown in Fig. 1. Consensus sequences of the HLA-A and -B alleles (Arnett and Parham, 1995) are shown on the top of the column. HLA-A2402 and -A2601 were sequenced from both the KE-4 CTL and tumor cells. HLA-B40012 cDNA was sequenced from only the KE-4 CTL line. Neither HLA-cw0102 nor-Cw0302 cDNA was sequenced from either one. 
cells was measured as reported (Seki et al. 1997). In brief, 20,000 tumor cells were added to a well of 96-well flatbottom micro plate in $100 \mu \mathrm{l}$ of RPMI1640 medium containing $10 \%$ heatinactivated FCS, and were incubated at $37^{\circ} \mathrm{C}$ for $12 \mathrm{hs}$, followed by addition of effector CTL at different effector to target cell $(\mathrm{E} / \mathrm{T})$ ratios. After an $18 \mathrm{hs}$ incubation, the supernatants were collected and assayed for cytokine production using the IFN- $\gamma$ ELISA (Genzyme Corp., Cambridge, MA). The limit of sensitivity of this ELISA was $5 \mathrm{pg} / \mathrm{ml}$.

Sequencing of HLA-class I alleles of the KE-4 CTL and tumor cells

HLA-class I serotypes of this patient were HLA-24/26, B54(22)/60(40), and Cw1/Cw3 by the conventional method (anti-HLA sera and Terasaki plate) with the PBMC. HLA-class I genotypes of the KE-4 patient were HLA-A2402/2602, B51/55 by the SSOP method with genomic DNA of the KE-4 tumor cells as reported (Nakao et al. 1995). HLA-C genotypes were $\mathrm{Cw0102/0302}$ by the restrction fragment length polymorphism (RFLP) with mRNA of the KE-4 tumor (Seki et al. 1997). HLA-class I cDNA were isolated from both the KE-4 CTL line and KE-4 tumor cells to confirm the expression of HLA-class I alleles on the CTL and tumors at the mRNA level. Briefly, $1 \times 10^{7}$ cells of either CTL or tumor cells were used to prepare mRNA using a Quick Prep mRNA Purification Kit (Pharmacia, Uppsala, Sweden), and first-strand cDNA was synthesized using a Super Script Preamplification Kit (BRL, Gaithersburg, MD). HLA-A specific oligonucleotides (5'-primer; GAATCTCCCCAGACGCCGA, 3'-primer; CACAGGTCAGCGTGGGAAG) and PCR were used to amplify HLA-A cDNA. HLA-B specific oligonucleotides (5'-primer; GAATCTCCCCAGACGCCGA, 3'-primer; AAACACAGGTCAGCATGGGA AC) or HLA-C specific oligonucleotides (5'-primer; GAATCTCCCCAGACGCCGA, 3'-primer; GAACACAGGTCAGTGTGGGG) and PCR were used to amplify HLA-B or -C cDNA, respectively. Each of these HLA-cDNA was cloned using an Eukaryotic TA Cloning Kit (Invitrogen, San Diego, CA) according to the manufacturer's instruction, and were sequenced using an A.L.F. DNA Sequencer (Pharmacia) as reported (Nakao et al. 1995). The part of the sequences was shown in Fig. 1. HLA-B40012 cDNA was sequenced from the KE-4 CTL line. HLAA2402 and -A2601 cDNA were sequenced from both the KE-4 CTL and KE-4 tumor cells. HLA-B5102, -Cw0102 or -Cw0302 cDNA was not sequneced from either one. The results suggest that HLA-A2402 and -A2601 were at least expressed on the KE-4 tumor cells at the mRNA level.

\section{Results and Discussion}

Fifty-two KE-4 CTL sublines were established from the parental KE-4 CTL line, and were tested for their IFN- $\gamma$ production by recognition of both the autologous KE-4 tumor cells and the allogenic tumor cell lines that shared only one of the HLA class I alleles with the KE-4 tumor cells. Namely, the following four tumor cell lines were used for the study; SW620 adenocarcinoma (A0201/2402, B0702/1518, Cw0702/0704), MKN-28 adenocarcinoma (A3101, B5101, Cw0304), OT testicular tumor (A0206, 
TABLE 1.

Recognition of tumor cells with different HLA-class I alleles by KE-4 CTL sublines ${ }^{a}$

\begin{tabular}{crrrrrrr}
\hline \multirow{2}{*}{$\begin{array}{c}\text { KE-4 CTL } \\
\text { subline }\end{array}$} & \multicolumn{6}{c}{ IFN- $\gamma$ production (pg/ml) by recognition of tumor cells } \\
\cline { 2 - 7 } & None & VA13 & KE-4 & $\begin{array}{c}\text { SW620 } \\
\left(\mathrm{A} 2402^{+}\right)\end{array}$ & $\begin{array}{c}\text { MKN-28 } \\
\left(\mathrm{B} 5101^{+}\right)\end{array}$ & $\begin{array}{c}\text { OT } \\
\left(\mathrm{B} 40012^{+}\right)\end{array}$ & $\begin{array}{c}\text { OSC20 } \\
(\mathrm{CW0102})\end{array}$ \\
\hline $128-11$ & 452 & ND $^{\mathrm{b}}$ & 1259 & 260 & 995 & ND & 344 \\
$128-14$ & 169 & ND & 461 & 134 & 212 & ND & 171 \\
$128-20$ & 252 & ND & 565 & 261 & 240 & ND & 514 \\
$64-2$ & 20 & 36 & 120 & 72 & 28 & 0 & 27 \\
$64-5$ & 25 & 17 & 71 & 13 & 15 & 7 & 17 \\
$32-10$ & 72 & 383 & 1064 & 3610 & 3192 & 0 & 701 \\
$32-13$ & 7 & 57 & 60 & 46 & 24 & 1 & 24 \\
$32-23$ & 14 & 10 & 29 & 10 & 7 & 0 & 9 \\
$32-29$ & 7 & 34 & 56 & 74 & 15 & 8 & 24 \\
$32-38$ & 214 & 262 & 1842 & 1275 & 1480 & 5 & 159 \\
$16-1$ & 110 & 98 & 780 & 83 & 130 & 0 & 78 \\
$16-18$ & 0 & 6 & 1228 & 24 & 0 & 0 & 8 \\
$16-27$ & 59 & 70 & 387 & 262 & 251 & 0 & 55 \\
$16-34$ & 38 & 22 & 772 & 44 & 31 & 0 & 0 \\
$8-1$ & 58 & 47 & 68 & 68 & 73 & 0 & 30 \\
$8-9$ & 41 & 55 & 146 & 98 & 262 & 0 & 40 \\
$8-11$ & 83 & 54 & 778 & 58 & 44 & 0 & 59 \\
$4-9$ & 28 & 181 & 1127 & 777 & 1436 & 0 & 142 \\
$4-10$ & 47 & 193 & 1713 & 940 & 1692 & 0 & 249 \\
\hline
\end{tabular}

a. KE-4 CTL sublines $\left(8 \times 10^{4}\right.$ cells/well) were incubated with the target cells $\left(8 \times 10^{4}\right.$ cells/well) shown above for $18 \mathrm{hs}$ followed by harvesting the cell-free supernatants. The supernatants were measured for their IFN- $\gamma$ activity by the IFN- $\gamma$ ERISA. The limit of the sensitivity was $10 \mathrm{pg} / \mathrm{ml}$.

b. Not done

B4001/3501, Cw0304), and OSC20 adenocarcinoma (A2/11, B4601, Cw0102). A matched HLA-class I allele was underlined. VA 13 fibroblast cell line that did not express HLA-class I mRNA was used as a negative control (Nakao et al. 1995). The representative results are shown in Table 1. A subline 128-11 produced higher levels of IFN- $\gamma$ by recognition of the KE-4 and MKN-28 tumors, but not the other tumors, suggesting that this subline recognized a shared tumor antigen presented by HLA-B5101 allele. A subline $128-14$ or $128-20$ produced higher levels of IFN- $\gamma$ by recognition of the KE-4 alone or both the KE-4 and OSC20 tumors, respectively. Therefore, the former might recognize either a shared tumor antigen expressed on HLAA2601 or an individual tumor-specific antigen presented by one of five HLAclass I alleles of KE-4 tumor cells. The latter might recognize a shared tumor antigen presented by HLA-Cw0102 allele. 


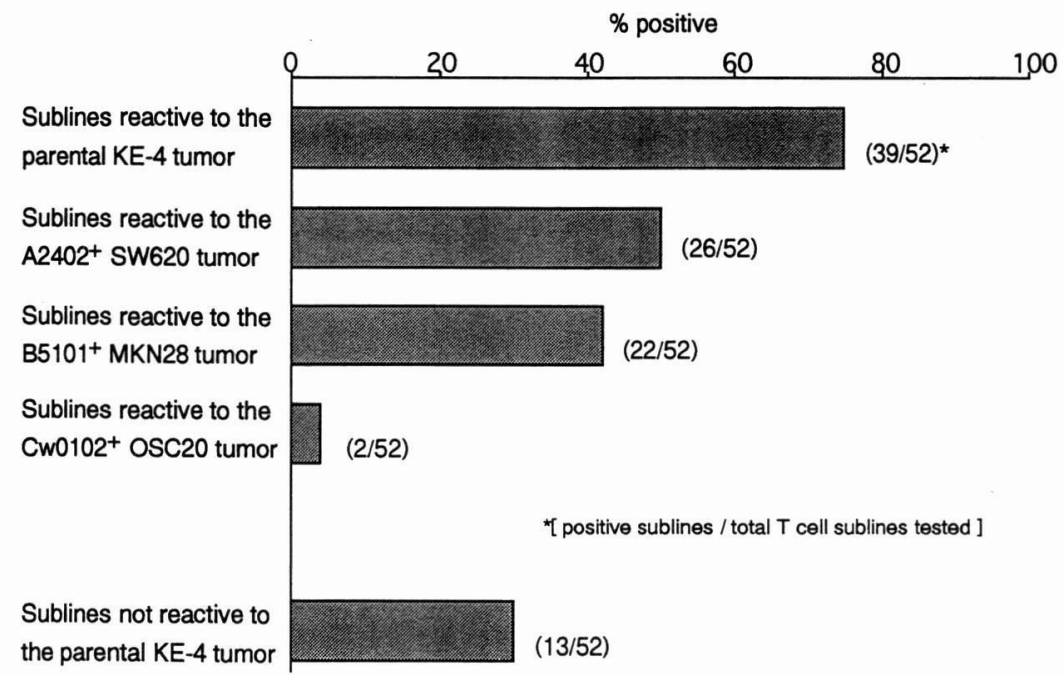

Fig. 2. Frequency of the KE-4 CTL sublines reactive to tumor cells with plural HLA-class I alleles: Fifty-two KE-4 CTL sublines were established from the parental KE-4 CTL line, and were tested for their IFN- $\gamma$ production by recognition of both the autologous KE-4 tumor cells and the allogenic tumor cell lines that shared only one of the HLA class I alleles with the KE-4 tumor cells. Namely, the following four tumor cell lines were used for the study; SW620 adenocarcinoma (A0201/2402, B0702/1518, Cw0702/0704), MKN-28 adenocarcinoma (A3101, B5101, Cw0304), OT testicular tumor (A0206, B4001/3501, Cw0304), and OSC20 adenocarcinoma (A2/11, B4601, Cw0102). A matched HLAclass I allele was underlined. VA 13 fibroblast cell line that did not express HLAclass I mRNA was used as a negative control (Nakao et al. 1995). The representative results are shown in Table 1. Fifty-two sublines were subdivided based on their patterns of reactivity to the above tumor cell lines, and the frequency of the CTL sublines reactive to tumor cells with different HLA-class I alleles are shown in Fig. 2.

A subline 64-2 produced higher levels of IFN- $\gamma$ by recognition of both KE- 4 and SW620 tumor cells, but not the others, suggesting that this subline recognized a shared tumor antigen presented by HLA-A2402 allele. In contrast, the level of IFN- $\gamma$ produced by a subline $32-13$ $(60 \mathrm{pg} / \mathrm{ml})$ by recognition of the $\mathrm{KE}-4$ tumor was not much different from that in response to VA13 cells (the negative control, $57 \mathrm{pg} / \mathrm{ml}$ ), suggesting that this subline did not recognize autologous tumor cells.

Fifty-two sublines were subdivided based on their patterns of reactivity to the above tumor cell lines, and the frequency of the CTL sublines reactive to tumor cells with different HLA-class I alleles are shown in Fig. 2. Thirty-nine of 52 (75\%) sublines produced IFN- $\gamma$ by 


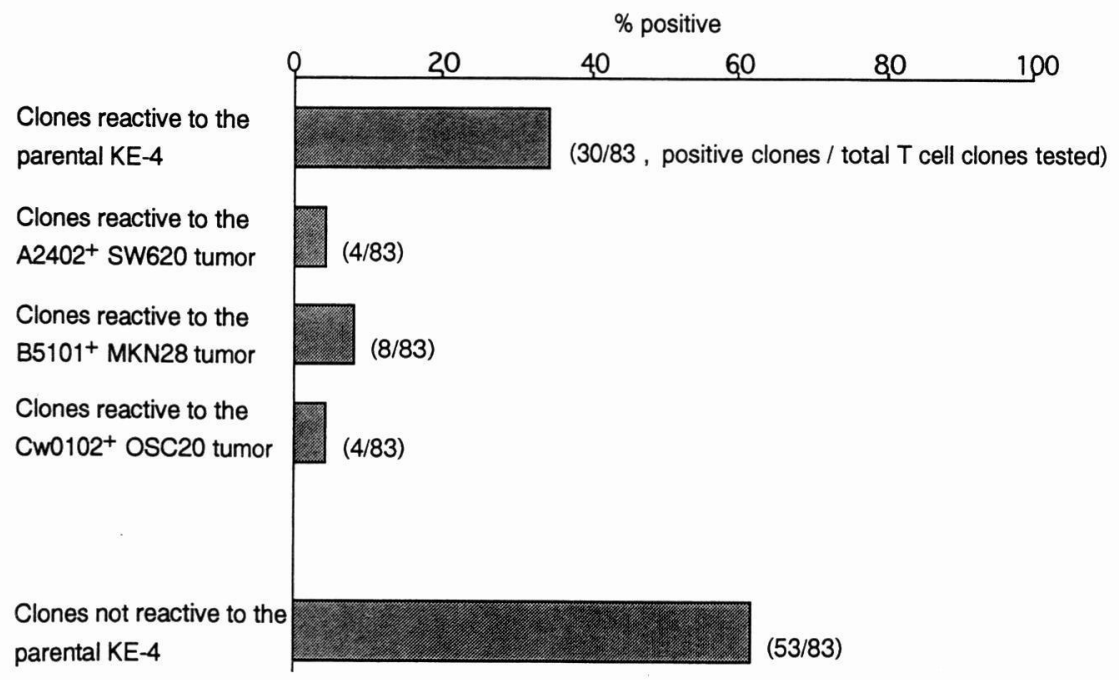

Fig. 3. Frequency of the KE-4 CTL clones reactive to tumor cells with plural HLA-class I alleles: Eighty-three KE-4 CTL clones were established were tested for their IFN- $\gamma$ production by recognition of the autologous KE- 4 tumor and the allogenic tumor cell lines shown in Table 1 . These 83 clones were subdivided based on their patterns of reactivity to the tumor cell lines. The frequency of the CTL clones reactive to tumor cells with different HLA-class I alleles are shown in Fig. 3.

recognition of the autologous $\mathrm{KE}-4$ tumor cells, whereas the other 13 failed to do. Twenty-six, 22, and 2 of these 39 CTL sublines also recognized the HLA$\mathrm{A} 2402^{+},-\mathrm{B} 5101^{+}$, and $\mathrm{Cw} 0102^{+}$allogenic tumor cells, respectively. These results suggest that the parental KE-4 CTL line recognizing shared tumor antigen(s) on HLA-A2601 allele (Nakao et al. 1995) also contains $\mathrm{T}$ cells recognizing shared tumor antigens presented by HLA-A2402 or -B5101 allele. A small number of $\mathrm{T}$ cells recognizing a shared tumor antigen presented by HLA-Cw0102 might also be present in the parental CTL line.

This study failed to show CTL reactive to a shared tumor antigen on HLA-B40012. Deletion of HLA-B40012 allele from the KE-4 tumor cells could be in part responsible to this failure. Alternatively, since only one HLAB40012 ${ }^{+}$testicular tumor cell line was eligible for this study, the other tumor may possess a shared tumor antigen with the KE-4 tumor presented by HLAB40012 allele.

Eighty-three KE-4 CTL clones were established to confirm the results with the sublines, since sublines shall consist of the mixture of $\mathrm{T}$ cells with different functions. These 83 clones were tested for their IFN- $\gamma$ production by recognition of the autologous KE-4 tumor and the allogenic tumor cell lines, and were subdivided based on their patterns of reactivity to the tumor cell lines. The 
frequency of the CTL clones reactive to tumor cells with different HLA-class I alleles are shown in Fig. 3. Thirty of 83 (36\%) clones produced IFN- $\gamma$ by recognition of the autologous KE-4 tumor cells, whereas the other 53 failed to do. Four, 8, 4 CTL clones recognized the HLA-A2402 ${ }^{+}, \mathrm{B} 101^{+}$, and Cw0102 ${ }^{+}$allogenic tumor cells, respectively. These results showed that the parental KE-4 CTL line consisted of 50 to $55 \%$ of T cells not reactive to the autologous tumor cells, 30 to $40 \%$ of $\mathrm{T}$ cells recognizing tumor antigen(s) presented on HLAA2601, and 10,5 , and $5 \%$ of $\mathrm{T}$ cells reactive tumor antigens presented on HLA-B5101, -A2402, and -Cw0102 alleles, respectively.

These results suggest that the parental KE-4 CTL line mainly consisted of $\mathrm{T}$ cells recognizing the shared tumor antigen on HLA-A2601 allele, which is in agreement with the results of the previous report (Nakao et al. 1995). These results also suggest that the parental KE$4 \mathrm{CTL}$ possesses a small number of $\mathrm{T}$ cells recognizing shared tumor antigen(s) presented by HLA-A2402, -B5101, or -Cw0102 allele. The discrepancy of the frequency of HLA-2402 or B5101restricted $\mathrm{T}$ cells between the subline and clone levels may be explained if the sublines mainly consisted of the HLAA2601-restricted $\mathrm{T}$ cells and a small number of HLA-A2402 or -B5101restricted $\mathrm{T}$ cells. The discrepancy of the frequency of $\mathrm{T}$ cells reactive to the autologous tumor versus that not reactive to the autologous tumor between the subline and clone level may also be explained if the subline consisted of both CTL reactive to the autologous tumor cells and non-CTL.
This study employed the IFN- $\gamma$ production assay, while the previous study used the ${ }^{51} \mathrm{Cr}$-release assay (Nakao et al. 1995). None of the SW620, MKN-28, OSC20 adenocarcinoma cells, or the OT testicular tumor cells were susceptible the lysis by the parental KE-4 CTL line or the CTL sublines tested (data not shown). The results suggest that IFN- $\gamma$ production assay is more sensitive for detection of CTL reactive to tumor antigens presented on HLA-class I allele of tumor cells.

We previously showed the presence of shared tumor antigen(s) on HLAA2601 allele recognized by the parental KE4-CTL (Nakao et al. 1995), and have recently identified a new gene encoding it's tumor antigen (Shichijo et al. unpublished results). We have now investigated the presence of shared tumor antigen(s) on HLA-A2402 allele recognized by the parental KE4-CTL. For this study, one of the HLA-A2402-restricted subline 4-5 was tested for their reactivity to a large panel of HLA-A2402+ solid tumors with different types of histology (Fig. 4). All these tumor cells shared only HLAA2402 allele with the KE-4 tumor cells. The 4-5 subline produced higher IFN- $\gamma$ by recognition of 4 (KE-4, YES-4, YES-6, and SKG-1) of 7 SCC, 2 (SW620, YPK1) of 4 adenocarcinomas, 1 (KMC1) of 2 hepatomas, and 2 (KOS-1, G292) of 3 osteosarcomas, and 1 transitional cell carcinoma (HT1376). The levels of IFN$\gamma$ by this subline in response to HLAA2402 ${ }^{+}$tumors (KE-4, KE-3) were dependent on the increased number of effector cells (Fig. 5). These results suggest that CTL subline 4-5 recognized tumor antigen(s) that is widely shared by different types of solid tumors and is 


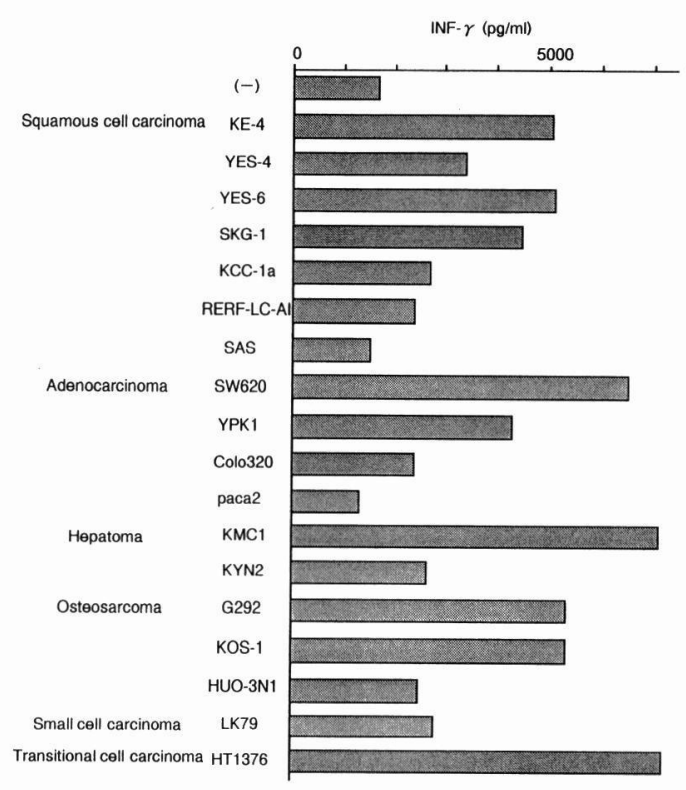

Fig. 4. Recognition of the HLA-A2402+ tumor cells by the CTL subline 4-5. I: An HLA-A2402-restricted subline 4-5 established from the parental KE-4 CTL line was tested for their reactivity to a large panel of HLAA2402+ solid tumors with different types of histology. All these tumor cells shared only HLA-A2402 allele with the KE-4 tumor cells. Levels of IFN- $\gamma$ produced by the 4-5 subline by recognition of the tumor cells are shown.

presented by HLA-A2402 allele.

In summary, this manuscript showed existence of CTL recognizing shared tumor antigens of SCC presented by plural HLAclass I alleles in a patient with esophageal cancer. This information shall be important for a better understanding of immune recognition of autologous tumor cells in cancer patients with SCC.

Acknowledgments: We thank Dr. Kaneshige (Shionogi Pharmaceutical Co.) for genotyping of HLA-class I alleles with SSOP method.

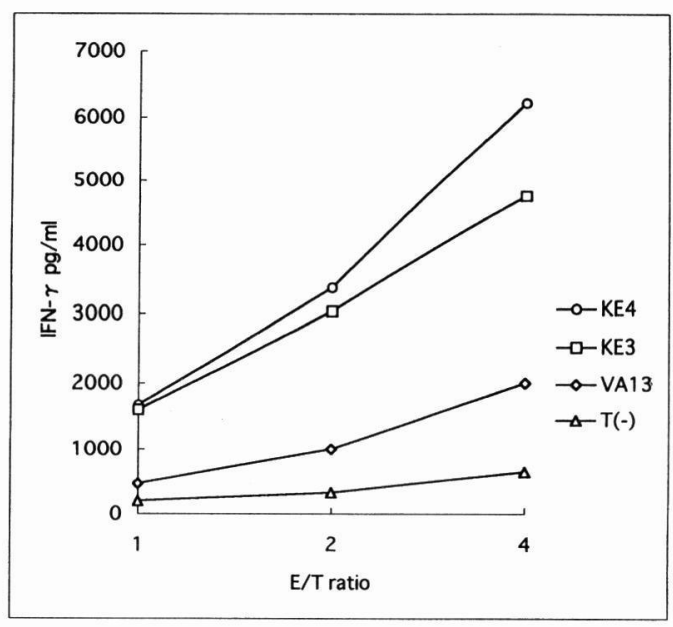

Fig. 5. Recognition of the HLA-A2402+ tumor cells by the CTL subline 4-5. II: The levels of IFN- $\gamma$ by the 4-5 subline in response to HLA-A2402+ tumors (KE-4, KE-3), VA13 cells, or by itself were tested at three different effectors to target cell ratios. The levels of IFN- $\gamma$ were dependent on the increased number of effector cells.

\section{References}

Arnett KL, and Parham P. HLA Class I nucreotide sequences, 1995. Tissue Antigens $1995 ; 45: 217-257$.

Boon T, Gajewski TF, and Coulie PG. From defined human tumor antigens to effective immunization? Immunol Today 1995; 16:334336.

Coulie GC, Brichard V, Van Pel A, Wolfel T, Schneider $\mathrm{J}$ et al. A new gene coding for a differentiation antigen recognized by autologous cytolytic $\mathrm{T}$ lymphocytes on HLA-A2 melanomas. J Exp Med 1994; 180:35-42.

Ioannides CG, Fisk B, Jerome KR, Irimura T, Wharton JT et al. Cytotoxic T cells from ovarian tumors can recognize polymorphic epithelial mucin peptides. J Immunol 1993; 151:3693-3703.

Itoh $\mathrm{K}$, Platsoucas $\mathrm{CD}$, and Balch $\mathrm{CM}$. 
Autologous tumor-specific cytotoxic T lymphocytes in the infiltrate of human metastatic melanomas: activation by interleukin 2 and autologous tumor cells, and involvement of the T cell receptor. J Exp Med 1988; 168:14191441.

Kawakami Y, Eliyahu S, Delgado CH, Robbins $\mathrm{PF}$, Rivoltini $\mathrm{L}$ et al. Cloning of the gene coding for a shared human melanoma antigen recognized by autologous $\mathrm{T}$ cells infiltrating into tumor. Proc Natl Acad Sci USA 1994a; 91:3515-3519.

Kawakami Y, Eliyahu S, Delgado $\mathrm{CH}$, Robbins $\mathrm{PF}$, Sakaguchi $\mathrm{K}$ et al. Identification of a human melanoma antigen recognized by tumor-infiltrating lymphocytes associated with tumor rejection. Proc Natl Acad Sci USA $1994 b ; 91: 6458-6462$.

Nakao M, Yamana H, Imai Y, Toh Y, Toh U et al.
HLA A2601-restricted CTLs recognize a peptide antigen expressed on squamous cell carcinoma. Cancer Res 1995; 55:4248-4252.

Peoples GE, Goedebuure PS, Andrews JVR, Schoof DD, and Eberlein TJ. HLA-A2 presents shared tumor-associated antigens derived from endogenous proteins in ovarian cancer. J Immunol 1993; 151:5481-5491.

Seki N, Hoshino T, Iwamoto O, Hayashi A, and Itoh K. HLA-A locus-restricted and tumorspecific CTL in tumor infiltrating lymphocytes of patients with lung cancer. Cell Immunol 1997; (in press)

van der Bruggen $\mathrm{P}$, Traversari $\mathrm{C}$, Chomez $\mathrm{P}$, Lurquin $\mathrm{C}$, de Plaen $\mathrm{E}$ et al. A gene encoding an antigen recognized by cytolytic $\mathrm{T}$ lymphocytes on human melanoma. Science 1991; 254:1643-1647. 